\title{
Proposal of a System for Estimating the Assembly Time in Small and Medium-Sized Enterprises
}

\author{
Štefan VÁCLAV, Albert MAREŠ, Stanislaw LEGUTKO*, Peter KOŠŤÁL, Daynier Rolando DELGADO SOBRINO
}

\begin{abstract}
This paper presents the results of the development of a specialized modifiable system for estimating the assembly time in practice. The proposal is mainly intended for small and medium-sized enterprises, which require the application of sophisticated and cost-effective solutions given the arising competitiveness in the assembly sector. The ATES system, i.e., Assembly Time Estimation System, which was created in Excel, was tested in the LEANLAB laboratory. The results correspond with current trends in this area and suggest directions for further development, especially in the field of 3D CAD systems and virtual reality.
\end{abstract}

Keywords: assembly; ATES; Microsoft Excel; time analysis; time estimation; video analysis

\section{INTRODUCTION}

The assembly process, as a part of the production process, is characterized by specific aspects to be considered in all phases and procedures relating to the design, operation, assembly sequence determination, ergonomics and rationalization of the assembled product and the entire assembly process [1-4]. In this regard one of the key concepts whose application is required and extremely useful in assembly is the so-called Lean Manufacturing. Within this framework, it is possible to target several problem areas, one of which is estimating assembly time. In this area, there are some methods and tools used for assembly time estimation. The implementation of these methods is characterized by several problematic aspects especially in small and medium-sized enterprises. The first aspect is the high price of the equipment or software to determine the time, especially when these are not on a regular basis. Using the software usually requires staff training; some methods such as MTM (Method Time Measurement) also require specialized training and examination.

Based on these issues, there is a clear need to create a relatively simple methodology that can work as a tool for assembly time estimation, which being user-friendly can be useable in the short and long term for a small or medium-sized enterprise. In this regard, the present paper introduces the Assembly Time Estimation System (ATES) which has been designed as part of a comprehensive package to determine the assembly time. This software application has been created in Excel, which is a tool that is commonly used in professional practice and does not usually pose difficulties when using it and applying the proposal to a given case study. The EXCEL application also ensures the possibility of modifying the ATES system for the specific conditions of the user.

\section{ANALYSIS OF METHODS AND TOOLS FOR ESTIMATING THE TIME OF ASSEMBLY PROCESSES}

The currently existing approaches for estimating the assembly time are mainly based on two basic types of methods. The first type does not need a real assembly workplace to determine the time of assembly. It is enough to know what and how it will be done in the assembly workplace. These methods work with the pre-determined time. It includes mainly methods like MTM [5, 6] or MODAPTS (Modular Arrangements of Predetermined Time Standards) [7], which in principle are based on a detailed analysis of the structure of the human movement. On this basis, using pre-determined times for different movements or actions, the total assembly time is calculated. The application of these types of methods requires specially trained staff. Because it is labourintensive, these methods come either in standalone specialized software packages, or are part of other specialized software packages. As an example, we can mention TASK Master 2000 from Eisbrenner Productivity [8] that addresses the MODAPTS analysis or TiCon from MTM Association for Research and Standards [9] that is based on the MTM analysis. Some software applications allow the selection of and/or offer the possibility for both MTM and MODAPTS. One example is the software module Time Estimation from Proplanner [10]. As mentioned above, these methods may be applied to determine the assembly time when the workstation does not exist yet. For example, when there is just a 3D CAD model of the workstation, it is possible to use these methods and the subsequent results serve as a basis for further balancing linked assembly workplaces. Furthermore, they can be used to analyse an existing assembly workstation, especially if the purpose is determining the assembly time, which will be the standard for the relevant workplace. These methods are also used, for example, for the analysis of the worker's movement structure in order to implement change or use the obtained data for calculating the assembly efficiency.

The second group of methods consists of methods dedicated to estimating the actual assembly time for an existing workstation i.e., and they need a real assembly workplace. In this case, it is possible, for example, to directly measure the assembly time in the workplace or to shoot a video and analyse the assembly according to it. The direct measurement of the assembly time may be most comfortable to realize using a stopwatch with the subsequent entry of data into forms. The disadvantage of this method is the human factor entering the measurement process. The main problem is breaking down the assembly operations into smaller operations usually not possible to identify in a precise manner. The need for measurement 
preparation is also an issue, i.e.: the division of the assembly into specific operations and movements, the selection of the workstation for measuring, the method for results processing, among others. Another solution approach is the use of special tools such as a TMU calculator [11], mobile touchpads, tablets, smartphones, and laptops and also a netbook from TimeStudyCompany [12], where software to determine the time is installed. It is also possible to use an automatic time recorder developed at the Fraunhofer Institute [13], which is placed on the worker's hand. These hardware solutions are usually a complement to an existing methodological procedure or software. For example, the software in equipment from TimeStudy is based on their software for video analysis; the TMU calculator uses the MTM method for time analysis.

As mentioned above, another possibility for estimating the assembly time is the use of video analysis. The base principle lies on the video capturing and subsequent investigation in a specialized software. Depending on the software type, the video analysis tool can be used for line balancing, cycle time setting and REFA analysis [14] TimeStudy software [12]. Also, for the preparation of instructions for workers, comparison of two videos Proplanner software [10] or DFX, FMEA analysis based on a video from the AVIX software that belongs to the Swedish company "Solme" [15].

In the particular case of fully automated assembly systems in the design process, the time can be determined by calculating the technical data of the specialized equipment manufacturers. Obtaining on-line information about the real assembly time is more comfortable in a fully automated system because some of the data from sensors can be used not only for assembly process control but also to determine the time of the particular assembly operation.

On the other hand, in the case of manual assembly operations which are equipped with sensors, it is possible to use the date from such sensors to estimate the time of several assembly operations or movements. These sensors are also usually used to control the process and workers themselves, e.g.: the presence of the components, the correct assembly operation ending, etc.

In the assembly process, there exist several levels for which it is necessary to define the time, or for which time data is the basis for extending activities. The first and lowest level deals with individual human movements. At this level, it is possible to use, for example, the MTM method or video analysis implemented in a specialized software that enables video slowdown while showing the actual time. In the second level, which relates to individual assembly operations, it is possible to use direct time measurement, video analysis, various specialized equipment, and also data from sensors. The third level relates to assembly time estimation for the workstation as a whole. Moreover, it is possible to compute the efficiency of the assembly process based on time data. It is possible to apply the same methods as in previous levels; in essence, this is the sum of times of either individual movements or assembly operations. In the fourth and final level relating to the assembly system, it is possible to use time data to determine cycle time, implement workplace balancing, and others.

\section{CONCEPT OF ASSEMBLY TIME ESTIMATION AND ANALYSIS}

Most of the authors of this paper worked on an international research project in 2013 called "Establishing LEAN knowledge and Laboratory". It was focused on lean assembly and, among other purposes, it also encompassed the development of methods and tools for both research and educational purposes. Based on several solved projects related to the assembly of automotive components in the frame of this laboratory LEANLAB, a requirement arose regarding the creation of a comprehensive methodological concept as well as a tool for actual estimation of assembly time. The identified basic needs and requirements focused on the following aspects:

- The concept should enable the optional application of several methods for exact assembly time estimation and analysis.

- The selected methods of assembly time estimation and analysis should cover all levels of detail-transactions, operations, workplace, and system.

- The concept should enable different types of data sources.

- The obtained data and information should be compatible with their mutual comparisons.

- In addition to time characteristics, the concept should also enable an analysis of structural aspects.

- Classification systems, time limits, and so on should be user-modifiable.

- The concept should allow the completion of other relevant methods and procedures such as the Yamazumi diagram, and others.

- The software tool itself should be user-friendly.

The model of assembly time estimation and analysis consists of three basic modules. Fig. 1 shows a schematic representation of this concept with levels of analysis, possible sources, and outputs. The first module is the video analysis which is based on a review of the recorded video of the activity of a worker and includes the possibility of slowing the video down, estimating time and classifying the worker's movement. For such an analysis, a special AOA (Assembly Operations Analysis) software like the one presented in [16] is required.

The second module is the ATES which aims at determining the duration of the worker's activity, in other words, it focuses on the estimation of the actual time directly at the assembly workstation. This methodology is based on the possibility of using video, web cameras or very direct monitoring of the assembly workstation.

On the other hand, on-line assembly analysis is a newly designed approach to obtain the assembly time of a workstation or several linked workstations based on five activities:

- The input of the assembled object at the workstation.

- Start of the assembly.

- Confirmation that the assembly process at the workplace or the assembled product is OK.

- End of the assembly operation.

- The output of the assembled product or assembled component from the workplace.

In the particular case of this paper, for creating the concept of on-line analysis it was necessary to take into account three essential requirements. The first requirement 
was the possibility to apply this model for three different modes of operation: on-line, off-line, and simulation. The second was that the model would work with other methods of assembly time estimation. The third requirement was that the model would have to be applicable for different types of manual assembly workplaces and therefore be maximally independent of the actual technical solution of the workstation both for one or several linked workstations of the final product or component assembly.

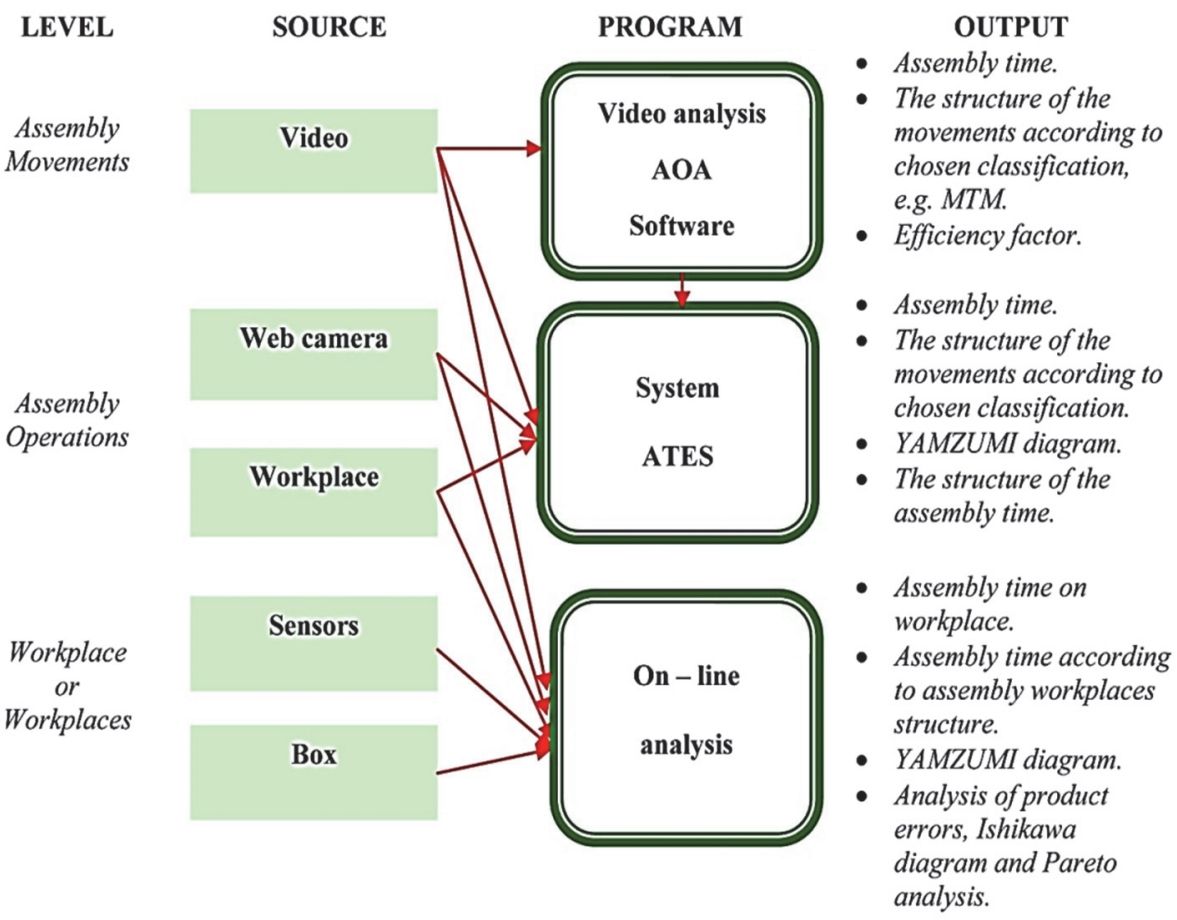

Figure 1 Model of the concept of assembly time estimation and analysis

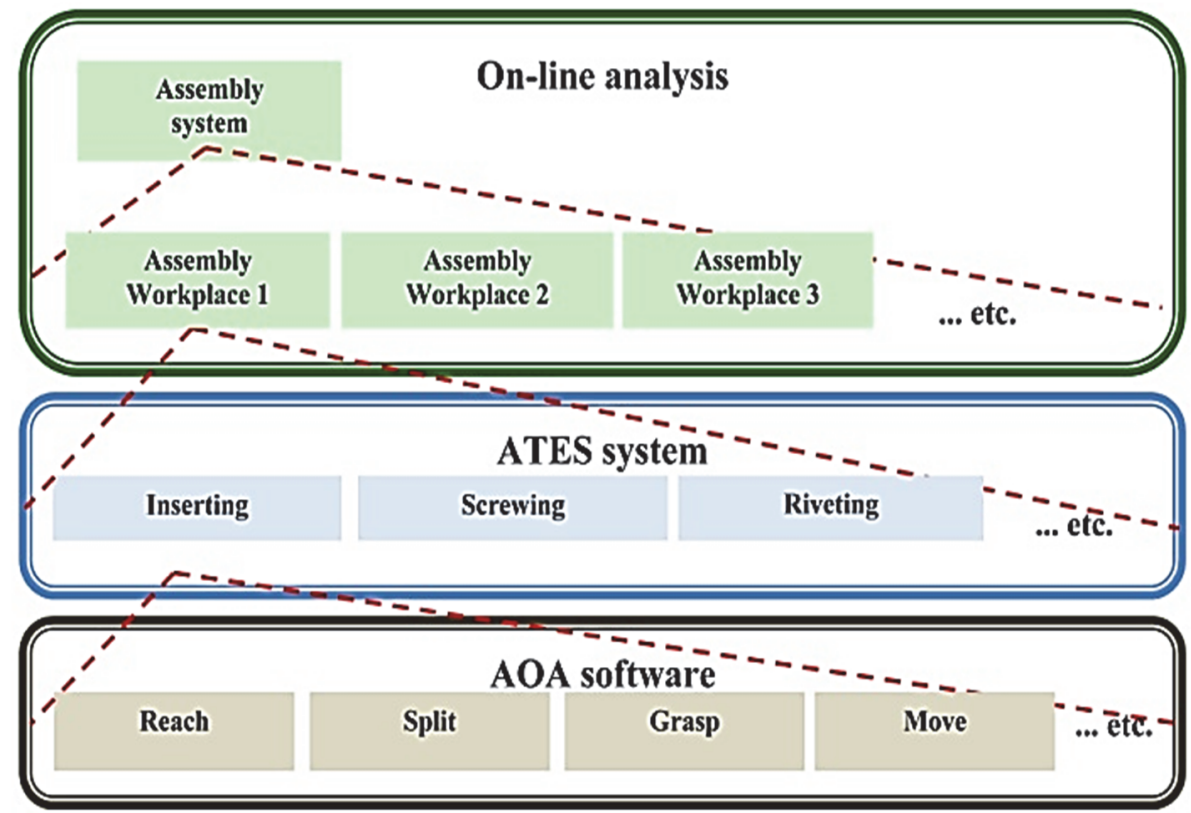

Figure 2 Areas of application areas of some assembly time estimation modules

The model also requires the entry of some additional parameters that characterize the layout of the workstation and the selected organization structure. It relates to the maximum supply between workstations expressed either as the number of batches or number of pieces, the maximum transport time for components from the previous workplace to the next workplace, and the maximum assembly time at each workstation. In addition to the time data from the five previously defined activities, the model also contains other additional data describing the structure of connected workplaces, the number of pieces in a batch, transport times, among others. The fundamental aspects and characteristics of this module were published in [17] and readers are encouraged to check this publication. Unlike the AOA software package and the ATES system, the on-line analysis assumes the long-term continuous monitoring of time characteristics.

In addition to time data, the presented approach also includes structural aspects. Besides the movement and operation duration or duration of the whole assembly in the 
workplace, the classification of individual activities is also essential. The proposed assembly time estimation system enables a different type of classification and then evaluation. The areas of application of the single basic modules are shown in Fig. 2.

\section{ANALYSIS OF THE ASSEMBLY OPERATION BASED ON THE VIDEO RECORDING}

During the solution of the project "Increasing productivity of hybrid assembly components in the automotive industry" and doctoral thesis [16], a software tool for the video analysing of the assembly process was developed. For the development of the software, the Microsoft Visual C\#.NET version 2003 programming language from the package Microsoft Visual .NET 2003 was chosen and used. A more detailed description of the software is given in [18]. The concept of the solution is in line with global trends and similar solutions. The software enables:

- To define the fundamental movements or operations as per their own needs.

- To follow the assembly process in detail (slow, stop, go back).

- To split the assembly time into sections and store these times and process them in further analysis.

During the analysis of assembly operation, it is necessary to pay attention, apart from to time characteristics, also to the ways of performing movements. The time data tells about the duration but says nothing about existing reserves or whether the movement was made in the most economical and efficient way.

If we use the philosophy that inefficient performance of movements is reflected as an extension of assembly time, it is possible to identify these inefficiencies either by comparing actual data with the data from another workstation where the same assembly operation is carried out, or by comparison with times defined under some of predetermined motion time methods, or by recording the same assembly process under changed conditions. This concept, as part of a comprehensive approach to the assembly time analysis, concerns the lowest level of detail, i.e., basic movements.

\section{THE ASSEMBLY TIME ESTIMATION SYSTEM (ATES)}

As mentioned before, the proposal is based on the direct determination of the assembly time. The created application for this proposal is based on the general requirements that are listed above and also the following ones:

- The need of stating the basic specification of the assembly process.

- The need of being able to read detailed data from AOA video analysis software.

- The possibility of taking into account the type variant option and the combination of data sources for obtaining time data.

- The possibility of being able to compare the results for one assembly process in the case these were obtained from different sources.

- Possible connection or system integration with other modules for supporting assembly process design.
The ATES system consists of the following functional modules:

- Module for assembly process specification.

- Module for evaluation of video analysis done using AOA software.

- Module for direct estimation of assembly time estimation at the workplace.

- Module for an estimate of assembly time based on the video.

- Module for an estimate of assembly time based on webcam monitoring.

- Module for YAMAZUMI diagram creation (Fig. 3).

- Module for a summary of results.

As an example, we can mention the analysis of motorcycle gearbox assembly, which was performed in the LEANLAB laboratory as part of the thesis [19]. The time analysis done was based on a captured video in AOA software and ATES system. A detailed assembly analysis was performed using the chosen MTM classification in the AOA software. Data from this in-depth analysis was loaded into the ATES system.

In this case the evaluation referred to the assembly time related to the worker movements and the efficiency coefficient calculated based on the specified limits according to the methodology and called primary and secondary times [20], and structure of movements for the right and left hand. Subsequently, the analysis of the same assembly process was also realized in the ATES system. The primary difference is in the detail of the investigation. The ATES system enables performing basic video analysis of assembly operations, reading, and entering the duration of process in the system. On the other hand, the AOA software allows slowing down the video, assign real-time for each defined movement and also add a selected type of classification. The analysis is detailed and difficult.The main characteristics of the AOA and ATES systems are shown in Tab. 1.

Table 1 The base characteristics of the AOA and ATES systems

\begin{tabular}{|c|c|}
\hline \multicolumn{1}{|c|}{ AOA software } & ATES system \\
\hline $\begin{array}{c}\text { Detailed analysis of the } \\
\text { movement of the right and left- }\end{array}$ & - Video analysis performed in \\
hand worker. & AOA program evaluate. \\
- Determining the time for every & factor of assembly. \\
movement. & Assembly operations analysis. \\
- Motion classification by the & - Determining assembly time \\
chosen classification system, & for every operation. \\
e.g., MTM, MODAPS, etc. & Compare results from both \\
- Creating a customized & studies. \\
classification of motions. & \\
\hline
\end{tabular}

The developed methodology, see Fig. 2, enables the performance of rapid analysis based on a video in the ATES system and subsequent return of detailed reports in the AOA software only for certain assembly operations. This approach saves time and enables the possibility of focusing on selected parts of the assembly that have problems or in which a change has been introduced, e.g.: a new jig, product design change, etc. Fig. 4 shows the print screen display module of ATES VIDEO. The work with module begins with importing the video of recorded assembly operations to excel sheet; there are Start and Stop buttons. Playing and stopping the video pushing the Start and Stop buttons automatically registers time data to the 
appropriate cell. Using these data it is possible to calculate the duration of operations. There is also a generated graph that shows the length and sequence of operation.

The other two modules of the ATES system are used for the direct measurement of assembly time at the workplace and analysis by monitoring a web camera. The main areas of application of this approach include:

- Evaluation of the impact of a product design change on assembly time and structure of assembly operation.

- Evaluation of the effect of changes to jig, tools, and storage on the assembly time and structure of assembly operation.
- Comparison of the various possible alternatives, whether of the whole assembly process or any part of it.

- Comparison of time data obtained in the design process, for instance, using the MTM method with realistic times obtained in the experimental workstation or directly in the production process.

- Video recording to support the education and training of new employees in the actual production process.

- Support for research and development activities focused, e.g., on creating own classifications of base assembly operations and movements with time data.

- Recording to identify assembly bottlenecks or for statistical purposes.
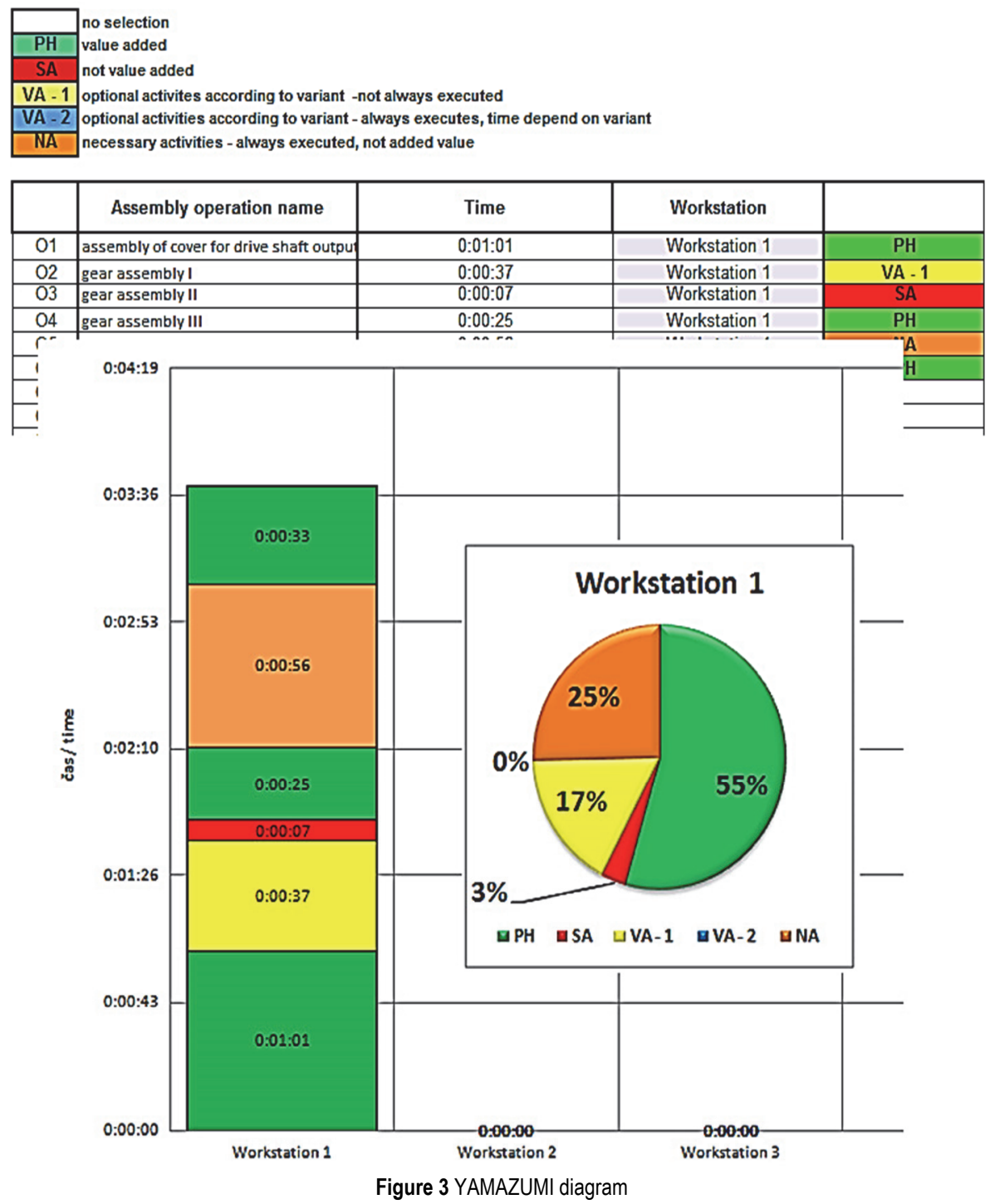

\section{APPLICATION OF THE METHODOLOGY TO A CASE STUDY AND DISCUSSION OF RESULTS}

The first activity when implementing the proposed ATES is to define the goals or purpose of analysis.

The second essential step is to become familiar with the assembly process so that errors cannot arise in dividing the assembly process into operations and errors in the time estimation process. If the analysis in the AOA software is selected, then it is also necessary to choose a classification system to be used or create one's own.
The worker who carries out the analysis must be familiar with the software, and if one of the methods requires training, the worker must have it. If the analysis is to be made on the basis of a video, it is necessary to record it and sometimes even modify it. Only then, it is possible using the system for time estimation. Once the analysis has been done, obtained data are to be interpreted, recommendations are to be possibly defined or even change may need to be implemented. 


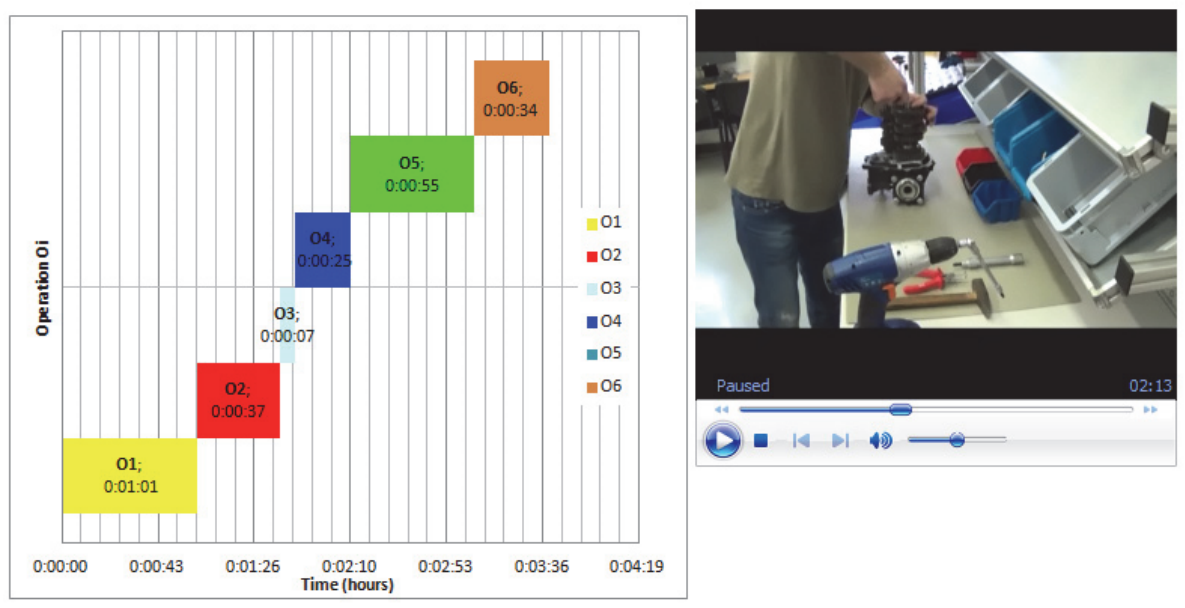

\begin{tabular}{|c|c|c|c|c|c|}
\hline \multirow{2}{*}{$\begin{array}{c}\text { Operation's } \\
\text { number }\end{array}$} & \multirow{2}{*}{ Assembly operation name } & \multicolumn{3}{|c|}{ Measuring 1} & \multirow[b]{2}{*}{ fre } \\
\hline & & from & to & Total Time & \\
\hline 01 & assembly of cover for drive shaft output & $0: 00: 00$ & 0:01:01 & 0:01:01 & \\
\hline $\mathrm{O} 2$ & gear assembly 1 & 0:01:01 & $0: 01: 38$ & 0:00:37 & \\
\hline $\mathrm{O} 3$ & gear assembly II & 0:01:38 & $0: 01: 45$ & 0:00:07 & \\
\hline$n a$ & man...nmmhlo, & n.n1.4k & $n \cdot n \cdot \cdot 1 n$ & n.nn:25 & \\
\hline
\end{tabular}

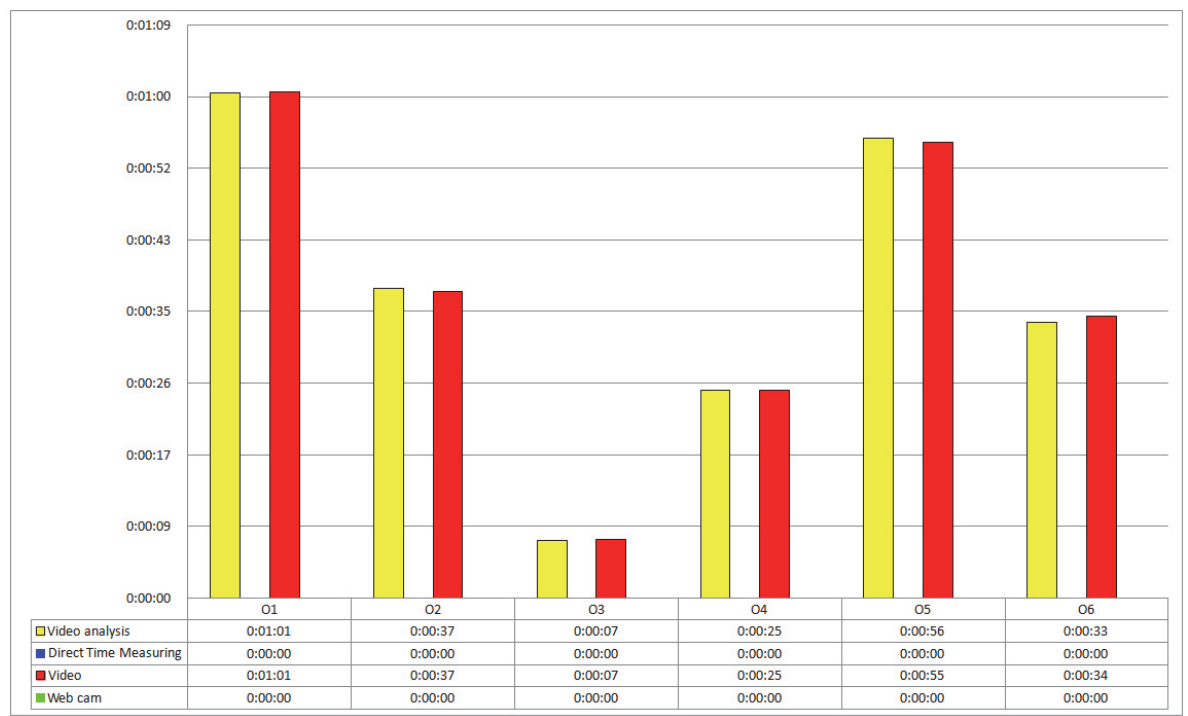

Figure 5 Result of comparison of assembly time estimation based on AOA software and ATES module

In this case study a Motorcycle gearbox assembly was first analysed based on the video in the AOA software. Six basic assembly operations were identified and then divided into movements of the right and left hand. After loading the data into the ATES system and specifying the limits for the primary and secondary analysis, it indicated that the total assembly time was 3 minutes and 39 seconds. The coefficient of efficiency for the left hand was $48 \%$ and $85 \%$ for the right hand. During the analysis in the AOA software, 130 movements were identified for each hand. For each movement, it was necessary to define time and movement classification. For comparison purposes, the same analysis was also implemented in the video module of the ATES system. The same video and of course, the same division into assembly operations were used. The sequence of assembly operations is known to significantly influence the assembly time and thus it was executed based on the previously defined assembly sequence for the given product. The total installation time is identical, e.g., 3 minutes and 39 seconds. The results appear graphically interpreted in Fig. 5.

Among the individual modules of the ATES system, some differences can be described as follows:

- The most labour-intensive analysis belongs to the AOA software. Other modules, i.e., analysis based on the video, direct measurement of time, or using a webcam are identical.

- Correcting mistakes is not problematic in the modules based on the video. In the case of direct measurement at the workstation and through the webcam, it is not possible to come back to already performed measurements. Correction can be achieved only by repeating the analysis of the same assembly process.

- The difficulties of the analysis process in terms of required knowledge about the assembly process itself are identical in all modules of the ATES system as well as the AOA software. However, the AOA software requires knowledge of the applied method for classification and working with the software itself. 


\section{CONCLUSION}

Based on the analysis made within this paper, and the results obtained, it can be stated that the solution to the presented problem of assembly time estimation is extremely flexible, and the results are adequate for standard practical applications. The main benefit of the proposed ATES system is its simplicity as part of a comprehensive assembly time determination package. The ATES software application was created in Excel, which enables wide and hassle-free applicability for practical needs.

The novelty of the proposed methodology lies in the design of a tool in the Microsoft Excel environment and the possibility to analyse in this application videos from an assembly operation (obtained by recording or directly from a web camera located in the workplace), and also on the possibility of executing direct time measurements. The proposed tool does not require any special know-how and is fast to work with. So far, we have not encountered in the states of the art and practice any tool to address this issue in the same way.

All essential attributes such as the number of workplaces in the module for creating YAMAZUMI diagrams, the number of measurements in the modules, the form of continuously updated graphs, etc. can be changed according to the user's requirements. Such a change is relatively simple because the ATES system is created in Excel. The ATES system does not require specialized training, and the possibility of its modification is a further significant advantage. As part of the presented concept, the modification of ATES for the needs of practice is now under testing. As part of the new development, attention is given to "on-line analysis" and also to aspects of the interconnection of time analysis with the systems for the simulation of assembly tasks. The fundamental question is whether the simulation results obtained in the CAD model correspond to real-time data collected in the presented ATES system. The ambition of the authors is to find these relationships and make recommendations for practical ways of interpreting the data obtained only in a virtual environment. Another promising area is the application of virtual reality resources, specifically the data glove in the process. In future research the authors plan to also publish the verification of assembly simulation using a real CAD model as well as the verification in practice conditions.

\section{Acknowledgments}

This paper was written thanks to the national KEGA Grant: 021STU-4/2018 - Development of a laboratory for the design and maintenance of production systems supported by the use of Virtual Reality.

\section{REFERENCES}

[1] Suszyński, M. \& Ciszak, O. (2019). Selection of Assembly Sequence for Manual Assembly Based on DFA Rating Factors. Advances in Manufacturing II, 45-47, Springer, Cham. https://doi.org/10.1007/978-3-030-18789-7_5

[2] Suszyński, M., Żurek, J., \& Legutko, S. (2014). Modelling of assembly sequences using hypergraph and directed graph. Technical Gazette, 21, 1229-1233
[3] Suszyński, M. \& Żurek, J. (2015). Computer aided assembly sequence generation. Management and Production Engineering Review, 6, 83-87. https://doi.org/10.1515/mper2015-0030

[4] Butlewski, M., Suszyński, M., Czernecka, W., Pajzert, A., \& Feniser, C. (2017). Ergonomic criteria in the optimization of assembly process. Proceedings of Matec Web of Conferences, 137, 424-431. https://doi.org/10.1051/matecconf/201713701015

[5] Karger, D., W. \& Bayha, F. H. (1987). Engineered Work Measurement: The Principles, Techniques, and Data of Methods-Time Measurement. 4th edition. New YorkIndustrial Press, 503 p. ISBN 978-0831111700

[6] Cieślak, R. \& Suszyński, M. (2017). Selection of methods used for analyzing the standard time needed to complete the assembly process by means of a fuzzy analytic hierarchy proces. Proceedings of Matec Web of Conferences, 137, paper 1014, 1-12, ClujNapoca. https://doi.org/10.1051/matecconf/201713701014

[7] Meyers, F. E. \& Stewart, J. R. (2001). Motion Time Study for Lean Manufacturing. $3^{\text {rd }}$ edition. Upper Saddle RiverPrentice Hall, , $370 \mathrm{p}$.

[8] TASK MASTER (2000). Dynamic Research Group. http://www.drshinnick.com/taskmaster-2000plus.html(10.01.2017).

[9] $\mathrm{Ti}$ Con. German MTM Association. https://www.dmtm.com/software/products/ticonforwindows /ticonbaseeng(10.01.2017)

[10] Time Estimation Proplanner. http://www.proplanner.com/en/products/protime_estimatio n (10.01.2017)

[11] TMU calculator. http://tmu-calculator.de/produkt (10.01.2017)

[12] Company Time Study. http://www.timestudy.de/hardwarezum-timestudy-t1-basismodul(10.01.2017)

[13] Fraunhofer Institute. http://www.iff.fraunhofer.de/content/ dam/iff/de/dokumente/publikationen/automatischezeiterfassung-fuer-manuelle-montageprozesse-fraunhoferiff.pdf (10.01.2017)

[14] REFA Bundesverband. http://refa-consulting.de/timetools (10.01.2017)

[15] Company Solme. http://www.avix.eu/en/our-products/avixmethod(10.01.2017)

[16] Mareš, A. (2006). Techniky skracovania času projektovania montážnych procesov a systémov (Time reduction techniques for assembly process and systems design doctoral thesis). Košice-SjF TU v Košiciach, 161.

[17] Senderská, K., Mareš, A., \& Zajac, J. (2012). Hardware of manual assembly workstation online analysis. Scientific Bulletin: Series D: Mechanical Engineering, 74(2), 103-110. http://www.scientificbulletin.upb.ro/rev_docs_arhiva/rezca 2 959535.pdf. https://doi.org/10.7562/SE2012.2.02.05

[18] Mareš, A. \&Senderská, K. (2006). Capturing video of assembly operations for analysis purpose. The $6^{\text {th }}$ International conference - Research and development in mechanical industry RADMI 2006. Budva (Montenegro) University of Kragujevac, Faculty of Mechanical Engineering of Kraljevo, 23-1-4. ISBN 86-83803-21-X

[19] Hegedüs, M. (2014). Analýza montáže vybraného komponentu pomocou videozáznamu (Selected component assembly analysis by using video recording - Diploma thesis). Košice-SjF TU v Košiciach. 62

[20] Lotter, B., Spath, D., \& Baumgartner, P. (2002). PrimärSekundär-Analyse. Kundennutzenmessung und Kundennutzenorientierung im Unternehmen. Renningenexpert-verlag, 124 ISBN 978-3-8169-2071-7 


\section{Contact information:}

Štefan VÁCLAV, Assoc. Prof.Ing., PhD

Institute of Production Technologies,

Faculty of Materials Science and Technology,

Slovak University of Technology in Bratislava,

J. Bottu 23, 91724 Trnava, Slovak Republic

E-mail: stefan.vaclav@stuba.sk

Albert MAREŠ, Ing. PhD

Faculty of Mechanical Engineering,

Technical University of Košice,

Masiarska 74, 04001 Košice, Slovak Republic

E-mail: albert.mares@tuke.sk

Stanislaw LEGUTKO, Prof. DSc. PhD. MSc. Eng.

(Corresponding author)

Faculty of Mechanical Engineering

Poznan University of Technology,

3 Piotrowostreet, 60-965 Poznan, Poland

E-mail: stanislaw.legutko@put.poznan.pl

Peter KOŠŤÁL, Assoc. Prof.Ing., PhD

Institute of Production Technologies,

Faculty of Materials Science and Technology,

Slovak University of Technology in Bratislava,

J. Bottu 23, 91724 Trnava, Slovak Republic

E-mail: peter.kostal@stuba.sk

Daynier Rolando DELGADO SOBRINO, PhD, MSc. Eng

Institute of Production Technologies,

Faculty of Materials Science and Technology,

Slovak University of Technology in Bratislava,

J. Bottu 23, 91724 Trnava, Slovak Republic

E-mail: daynier_sobrino@stuba.sk 